\title{
Milk yield and quality traits in different lactation stages of Damascus goats: Concentrate and pasture based feeding systems
}

\author{
Akın YAKAN ${ }^{1}$, Hüseyin ÖZKAN ${ }^{2}$, Aysel ERASLAN ŞAKAR² ${ }^{2}$ Cafer Tayyar ATEŞ ${ }^{3}$, \\ Necmettin ÜNAL ${ }^{4}$, Ömür KOÇAK ${ }^{5}$, Gökhan DOĞRUER $^{6}$, Ceyhan ÖZBEYAZ $^{4}$
}

\begin{abstract}
${ }^{1}$ Erciyes University, Veterinary Faculty, Department of Animal Breeding, Kayseri; ${ }^{2}$ Mustafa Kemal University, Veterinary Faculty, Department of Genetic, Hatay; ${ }^{3}$ Mustafa Kemal University, Veterinary Faculty, Department of Animal Breeding, Hatay; ${ }^{4}$ Ankara University, Veterinary Faculty, Department of Animal Breeding, Ankara; ${ }^{5}$ Istanbul University, Veterinary Faculty, Department of Animal Breeding, Istanbul; ${ }^{6}$ Mustafa Kemal University, Veterinary Faculty, Department of Obstetrics and Gynecology, Hatay,

Turkey.
\end{abstract}

Summary: This study aimed to survey milk yield, lactation stages and milk quality traits of Damascus goats reared under different feeding systems. Goats were divided according to feeding systems as pasture based and concentrate based. Feeding systems were found to have significant effect on lactation milk yield but not on lactation duration. Although differences between feeding systems were not found significant on $\mathrm{pH}$, milk composition factors (fat, lactose, dry matter), somatic cell count and malondialdehyde (except for early lactation stage) for each lactation stage, significant effects were detected on same parameters among lactation stages. Calcium levels at early lactation stage in milk differed either between feeding systems or among lactation stages. Also, other minerals were found to decrease with lactation. While most of the fatty acids were affected in any of the lactation stages by the feeding system, all of them were significantly altered by lactation stages. Pastured goats had a lower percentage of total saturated fatty acids, atherogenic index, thrombogenic index and odour index ratios. In accordance with milk fatty acid composition, compared to the milk obtained from goats fed with concentrate, the milk obtained from the goats that pastured was healthier and early lactation stage was found to produce healthier milk than late lactation stage.

Keywords: Damascus goat, feeding system, lactation stage, milk quality, milk yield.

\section{Şam keçilerinde laktasyonun farklı dönemlerinde süt verimi ve süt kalitesi özellikleri: Konsantre ve meraya dayalı besleme sistemleri}

Özet: $\mathrm{Bu}$ çalışma, farklı besleme sistemlerindeki Şam keçilerinin laktasyonun farklı dönemlerinde süt verimi ve süt kalite özelliklerinin araştırılması amacıyla yapılmıştır. Keçiler konsantre yeme ve meraya dayalı olarak iki farklı besleme sistemine ayrılmıştır. Besleme sistemlerinin, laktasyon süresi üzerine etkisi önemsiz olurken laktasyon süt verimi üzerinde önemli farklılığa sebep olmuştur. Besleme sistemleri arasında pH, süt kompozisyonu (yağ, laktoz, kuru madde), somatik hücre sayısı ve laktasyonun erken dönemleri hariç malondialdehit düzeyleri benzer olurken, aynı parametreler üzerinde laktasyon dönemlerinin etkisi önemli olmuştur. Sütteki Kalsiyum seviyeleri erken laktasyon dönemlerinde besleme sistemleri bakımından farklılık göstermiştir. Ayrıca laktasyon dönemleri arasında da farklılık meydana gelmiştir. Sütte bulunan diğer mineral maddelerin de laktasyonla beraber azaldığı tespit edilmiştir. Yağ asidi kompozisyonu besleme sistemlerinden büyük oranda etkilenirken, tüm yağ asitleri laktasyon dönemlerine göre önemli düzeyde farklılık göstermişlerdir. Meraya dayalı besleme sistemindeki keçilere ait sütler daha düşük toplam doymuş yağ asidi oranı, aterojenik indeks, trombojenik indeks ve koku indeks değerine sahip olmuştur. Süt yağ asidi kompozisyonu bakımından meraya dayalı beslenen keçilerden elde edilen süt konsantre yeme dayalı beslenen gruba göre daha sağlıklı bulunmuştur. Benzer şekilde erken laktasyon döneminde üretilen süt, geç laktasyon döneminde üretilene göre daha sağlıklı olmuştur.

Anahtar sözcükler: Besleme sistemi, laktasyon dönemi, süt kalitesi, süt verimi, Şam keçisi.

\section{Introduction}

Turkey goat population is approximately 11 million heads, only $2.5 \%$ of them is dairy. Most of dairy goats have been raised in Southern Turkey. Damascus goats are known as dairy and more adaptable to the environmental conditions. Guler et al (17) stated that lactation milk yield, duration, total solid and fat is $330.731,244.5$ days, $12.90 \%$ and $4.02 \%$, respectively.

Compared to cow and sheep milk; goat milk and goat products have less allergenic components and they generate higher economic gain; therefore, they are significant in human nutrition $(13,37)$. Increase in the 
number of goats in Turkey for the past years (43) has also shown that goat milk and its products are better valued and the value of goat milk has simultaneously increased as well. In the last years, quality parameters of milk have been taken into account as well as milk yield because quality parameters have been found important for consumer preference. Generally, in developing countries, high incidences of cardiovascular diseases are observed and clients demand top quality animal products with connatural flavor and taste (45). Similar to cow milk, goat milk delivers many nutrients with low energy content and it is advantageous for clients' well-being of throughout their lives. Therewithal, goat milk has ample benefits over cow and sheep milk since it can be used as an alimentary resource and remedial food for both infants and children (37).

Production systems or diet affects the composition of goat milk and goat milk products (14). Grazing environments of goats include a number of crucial metabolites that have advantages for human wellness and comprise alkaloids, fatty acids, tannins and flavonoids (13). On the other hand, milk composition may change during all lactation stages because of pasture or concentrate based feeding systems (23).

Community has grasped the significance of meadowgazing goats. European countries lately decreased amounts of grazing goats due to hardships of finding shepherds and the activity of grazing, lack of meadows, diminished capacity of soils and abundance of goat breeds with low grazing ability (45). Thence, it is significant to compare pasture or concentrate based feeding systems.

While milk yield is one of the most important parameters in dairy goat systems, milk quality parameters (chemical composition, fatty acid composition, lipid oxidation capacity, mineral matter, etc.) are also substantial. Moreover, pricing is affected by milk quality levels such as milk fat content, dry matter, $\mathrm{pH}$, etc.

This research intended to investigate milk yieldquality parameters and the changes observed during all lactation stages of Damascus goats under pasture based and concentrate based feeding systems.

\section{Material and Methods}

Ethical consideration: The actual research was approved by Animal Studies Ethic Committee of Mustafa Kemal University (Approval number: 2013/9-10).

Animal material and experiential procedures: The experiment was performed at a private goat farm in Hatay at south central region of Turkey. The animal materials of experiments were randomly selected from 400 heads flock. The goats and kids were medicated for the parasite with ivermectin/clorsulon and foksim and vaccinated against enterotoxaemia, foot and mouth diseases, ecthyma, agalaxia, mycoplasmosis, blue tongue and peste des pestits ruminants at physiologically suitable times. All goats were familiarized to the feed and pens for two weeks. All goats in the study were controlled against mastitis by using California Mastitis Test and mastitis negative goats were used in experiments and the goats never caught clinical mastitis during lactation. Experiments were carried out with concentrate-based feeding group (CBFG) $(n=18)$ and pasture-based feeding group (PBFG) $(n=18)$. Age of the goats in $C B F G$ and PBFG were $4.70 \pm 0.28$ ( 10 heads is $4 \leq$ ages and 8 heads is $4>$ ages) and $4.38 \pm 0.24$ ( 9 heads is $4 \leq$ ages and 9 heads is 4> ages), respectively $(\mathrm{P}>0.05)$ and parturition type was the same in both pasture based and concentrate based groups (6 single, 12 twin). The goats (4 single and 8 twin) used for milk mineral matter and fatty acid composition were randomly selected from each group. Goats in both feeding groups were sheltered in fattening pens $\left(4 \mathrm{~m}^{2}\right.$ of ground per goat) prepared for each group. While CBFG was housed all time in pen and fed $1.2 \mathrm{~kg} / \mathrm{goat} / \mathrm{day}$ concentrate feed and $1 \mathrm{~kg} / \mathrm{goat} /$ day wheat straw, PBFG went to pasture one week later from parturition for grazing between 06:00-18:00 each day and was housed in pen after grazing. The PBFG goats consumed $0.6 \mathrm{~kg} /$ goat/day the concentrate feed (Table 1). The kids of CBFG stayed with their mothers until weaning ( 3 months old), but in milk control days, kids were separated from their mothers after last milking and separated kids were fed with a bottle during control days. Similarly, PBFG kids were kept in pen until they were 1 month old and later, they went to pasture together with their mothers but didn't go to pasture at milk control days. Like CBFG, PBFG kids also were weaned when they were 3 months old. Briefly, the kids in both groups were isolated from their mothers for 24 hours before milk control. Lactations were generally continued between January-February and August-September in both groups.

Milking and milk quality analyses: All goats used for milk yield and milk quality were milked with an interval of 14 days by hand milking methods during lactation and when daily milk yield decreased lower than $100 \mathrm{~g}$ (a criterion that was accepted to be the end of lactation for goats). Although milk control stages were in 14-day intervals, all milk quality values were assessed for early lactation stage (ELS), mid lactation stage (MLS) and late lactation stages (LLS). While the point on lactation curve between the start of lactation and maximum milk yield level was accepted as ELS, other lactation areas on lactation curve were divided into two parts as MLS and LLS. All milk yields were normalized for each goat by interpolation methods and lactation milk yields were calculated according to Trapez II methods (46). 
Table 1. Chemical and physical composition of concentrate feeds

Tablo 1. Konsantre yemin kimyasal ve fiziksel kompozisyonu

\begin{tabular}{|c|c|c|c|c|c|}
\hline \multicolumn{2}{|c|}{ Items contents } & \multicolumn{2}{|l|}{ Proportions (\%) } & \multirow{2}{*}{$\frac{\text { Proteins }(\%)}{11.4}$} & Metabolic energy (kcal/ kg) \\
\hline Wheat & & 19.5 & & & 2800 \\
\hline Maize barn & & 20.6 & & 19.5 & 2670 \\
\hline Corn & & 18.5 & & 7.8 & 2880 \\
\hline Sunflower meal & & 15.5 & & 28.0 & 2570 \\
\hline Cottonseed meal & & 10.0 & & 31.0 & 2170 \\
\hline Barley & & 7.5 & & 10.8 & 2750 \\
\hline Wheat barn & & 2.3 & & 14.9 & 2320 \\
\hline Molasses & & 5.0 & & 8.5 & 2890 \\
\hline Marble powdere & $\left(\% 38 \mathrm{Ca}^{++}\right)$ & 0.3 & & - & - \\
\hline $\mathrm{NaCl}$ & & 0.7 & & - & - \\
\hline Premix* & & 0.1 & & - & - \\
\hline Total & & 100.0 & & 16.70 & 2649.28 \\
\hline Dry matter & & 88.91 & & & \\
\hline Crude ash & & 5.96 & & & \\
\hline Ether extract & & 2.58 & & & \\
\hline Crude protein & & 16.51 & & & \\
\hline \multicolumn{6}{|c|}{ Fatty acid composition (\%) } \\
\hline $\mathrm{C} 12: 0$ & 0.14 & C18:1 & 21.14 & $\mathrm{C} 22: 1$ & 1.49 \\
\hline C14:0 & 0.24 & C18:2 n6 & 53.48 & C22:6 n3 & 0.18 \\
\hline $\mathrm{C} 15: 0$ & 0.09 & $\mathrm{C} 18: 3 \mathrm{n} 3$ & 3.52 & $\mathrm{C} 24: 0$ & 0.32 \\
\hline C16:0 & 15.19 & C20:0 & 0.41 & $\sum$ SFA & 18.96 \\
\hline C16:1 & 0.32 & C20:1 & 0.91 & $\sum$ MUFA & 23.86 \\
\hline C18:0 & 2.22 & C22:0 & 0.35 & $\sum$ PUFA & 57.18 \\
\hline
\end{tabular}

*: Per $1.5 \mathrm{~kg}$ premix contains15 $000000 \mathrm{IU}$ Vit A, $3000000 \mathrm{IU}$ Vit $\mathrm{D}_{3}, 50000 \mathrm{IU}$ Vit E, $50 \mathrm{~g}$ manganese, $50 \mathrm{~g}$ ferrous, $50 \mathrm{~g}$ zinc, 10 $\mathrm{g}$ copper, $0.8 \mathrm{~g}$ iodine, $0.2 \mathrm{~g}$ cobalt, $0.3 \mathrm{~g}$ selenium.

*: Her 1.5 kg'llk premix 15000000 IU Vit A, 3000000 IU Vit D3, 50000000 IU Vit E, 50 g Manganez, 50 g Demir, 50 g Çinko, 10 g Bakır, 0.8 g İyot, 0.2 g Kobalt, 0.3 g Selenyum içermektedir.

On control days, milk samples of approximately 200 $\mathrm{ml}$ were taken from each goat for milk quality analyses and these milk samples were swiftly transported to laboratory under ice bath. While $\mathrm{pH}$ and electric resistance were determined with a portative $\mathrm{pH}$ meter (HI83141, Hanna Ins.), milk color was colorimetrically tested as $\mathrm{L}^{*}$ (lightness), $\mathrm{a}^{*}$ (redness) and $\mathrm{b}^{*}$ (yellowness) index values (Konica-Minolta CR-400). Before color analysis, reflection photometer was already calibrated with a blank and later, $20 \mathrm{ml}$ of the samples were added to transparent plastic bags fitted to the photometer output. Readings were replicated three times per sample.

Forty $\mathrm{ml}$ milk samples with chemical tablets (Microtab II, Weber Sci.) were allocated for fat, protein, lactose, dry matter and somatic cell count (SCC) and later analyzed for milk chemical component (Combi 150, Bentley Ins.), SCC (Somacount 150, Bentley Ins.).

Malondialdehyde analysis (MDA) and milk mineral matter analyses were made from frozen milk samples that were kept at $-24^{\circ} \mathrm{C}$ until analysis. While MDA levels were determined with UV-Spectrophotometers based on Esterbauer \& Cheeseman (12), mineral matters were analyzed by Microwave Plasma Atomic Emission
Spectroscopy (MP-AES 4100, Agilent Tech.). For mineral matters ( $n=12,4$ single and 8 twin parturition), milk samples were burned under nitric acid + perchloric acid first and later analyzed at suitable dilutions at the following device conditions: uptake time $30 \mathrm{~s}$; rinse time $15 \mathrm{~s}$; stabilization time $25 \mathrm{~s}$; pump speed $15 \mathrm{rpm}$; wave length and nebulizer pressure for $\mathrm{Ca}, \mathrm{Mg}, \mathrm{Fe}, \mathrm{Cu}, \mathrm{Zn}$ were 393.366, 285.213, 371.993, 324.754, $213.857 \mathrm{~nm}$ and 120 , 240, 120, 240, $140 \mathrm{kPa}$, respectively.

Two $10 \mathrm{ml}$ milk samples from each goat $(\mathrm{n}=12,4$ single and 8 twin parturition) were centrifuged for $15 \mathrm{~min}$, $3000 \mathrm{~g}$ at $+4{ }^{\circ} \mathrm{C}$ and milk cream from top of tube was gathered in $1,5 \mathrm{ml}$ vials, frozen and frozen at $-24{ }^{\circ} \mathrm{C}$ until analysis. During analysis, approximately $500 \mu \mathrm{l} \mathrm{milk}$ cream was saponified with $2 \mathrm{ml}$ of $2 \mathrm{~N}$ methanolic $\mathrm{KOH}$ for $2 \mathrm{~min} / \mathrm{mixed}$ at room temperature. Later, $4 \mathrm{ml} \mathrm{n}$-Heptan was added and mixed for 1 min. Later, tubes were centrifuged at $200 \mathrm{~g} / 3 \mathrm{~min}$ and allocated to separate organic phase. Fatty acid methyl esters (FAME) were collected from the top layer and transferred into vials. Separation of fatty acids was performed with HP Agilent 6890/5972 model GC-MS equipped with HP Innowax colon (60 m length, $0.25 \mathrm{~mm}$ i.d. x $0.25 \mu \mathrm{m}$ film). While 
120 Akın Yakan - Hüseyin Özkan - Aysel Eraslan Şakar - Cafer Tayyar Ateş - Necmettin Ünal - Ömür Koçak - Gökhan Doğruer Ceyhan Özbeyaz

injector temperature was set at $250{ }^{\circ} \mathrm{C}$, detector temperature was $270^{\circ} \mathrm{C}$. Injection was splitless with a total injection volume of $1 \mu \mathrm{l}$ and injector was washed three times with n-Heptan. Oven temperature was programmed initially at $120^{\circ} \mathrm{C}$ for $3 \mathrm{~min}$ and was increased to $250{ }^{\circ} \mathrm{C}$ with a $3{ }^{\circ} \mathrm{C} / \mathrm{min}$ ramp rate. Helium was used as a carrier gas.

Statistical analyses: All statistical analyses were performed using the SPSS 14.0 statistical package (license number: 9869264). Effect of feeding systems, age and birth types (fixed factors) based on the comparisons (between pasture and concentrate based groups; between $4 \leq$ and $4>$ ages; between single and twin birth type) on milk yield, lactation duration and milk quality traits were tested by Generalized Linear Models. On the other hand, effect of lactation stages on milk yield and milk quality characteristics was analyzed with repeated measurement One-Way ANOVA while Duncan's multiple range test was applied to evaluate the significance of the difference.

\section{Results}

While lactation curve for feeding systems is presented in Figure 1, mean values of lactation milk yield and lactation duration in groups are shown in Table 2. Lactation milk yield was $238.185 \mathrm{~kg}$ and it was higher in pasture-based group $(\mathrm{P}<0.001)$ and older age group $(\mathrm{P}<0.05)$ but no difference was found for lactation duration $(\mathrm{P}>0.05)$.

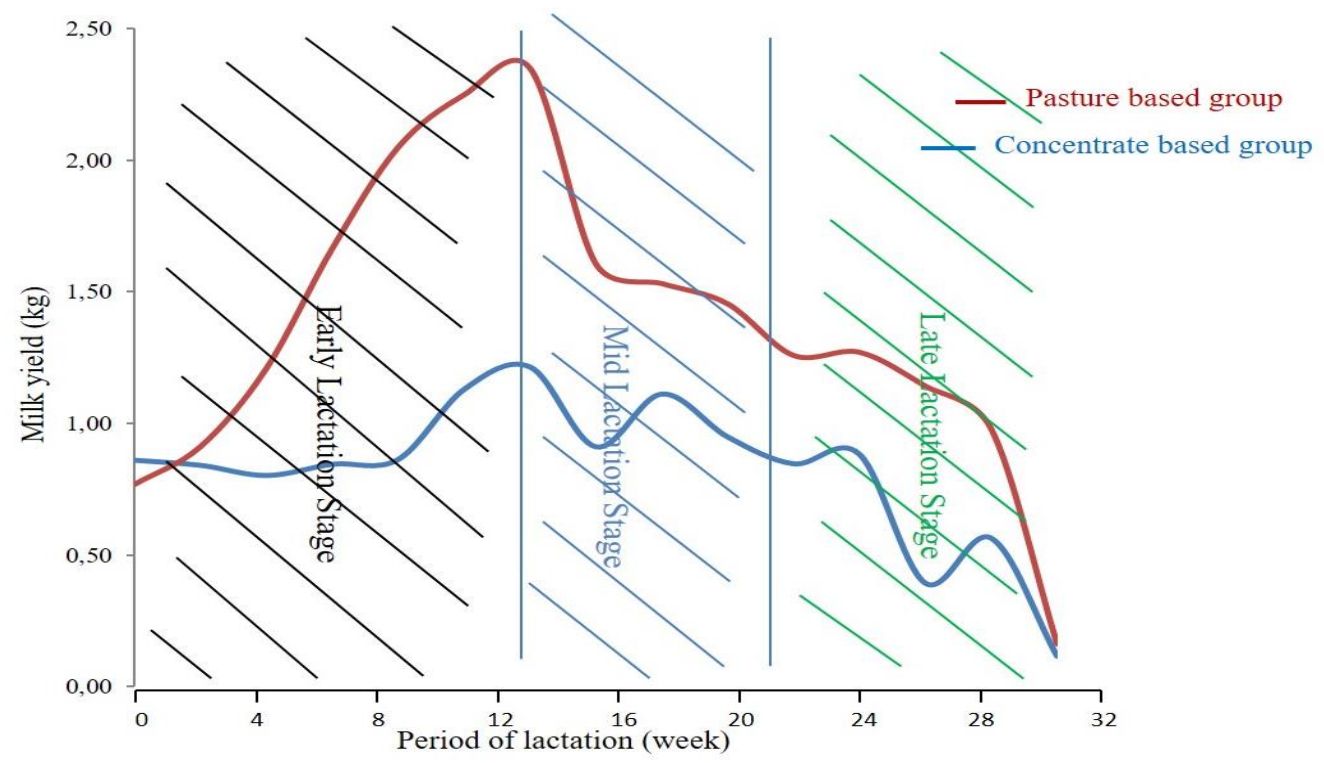

Figure 1. Lactation curves for feeding systems

Resim 1. Besleme sistemlerine göre laktasyon eğrileri

Table 2. Pooled Means \pm SE of milk yield and lactation duration for feeding systems, age and birth type

Tablo 2. Besleme sistemleri, yaş ve doğum tipi için süt verimi ve laktasyon süresine ait ortalamalar ve standart hatalar

\begin{tabular}{ccccc}
\hline \multirow{2}{*}{ Characters } & Pooled Means \pm SE & $\begin{array}{c}\text { Feeding systems } \\
\text { (Means difference }\end{array}$ & $\begin{array}{c}\text { Pge } \\
\text { (Means difference }\end{array}$ & $\begin{array}{c}\text { Birth type } \\
\text { (Means difference }^{\boldsymbol{k}} \text { ) }\end{array}$ \\
\hline Lactation milk yield (kg) & $238.185 \pm 10.06$ & $126.297^{* * *}$ & $-42.171^{*}$ & $-16.646^{-}$ \\
\hline Lactation duration (days) & $210.487 \pm 4.57$ & $16.184^{-}$ & $7.939^{-}$ & $-15.298^{-}$ \\
\hline ELS (kg/day) & $1.259 \pm 0.07^{\mathrm{a}}$ & $0.682^{* * *}$ & $-0.276^{*}$ & $-0.115^{-}$ \\
MLS (kg/day) & $1.239 \pm 0.06^{\mathrm{a}}$ & $0.569^{* * *}$ & $-0.187^{-}$ & $-0.050^{-}$ \\
LLS (kg/day) & $0.713 \pm 0.04^{\mathrm{b}}$ & $0.436^{* * *}$ & $-0.078^{-}$ & $0.027^{-}$ \\
P & $* * *$ & & & \\
\hline
\end{tabular}

ELS: Early lactation stage; MLS: Mid lactation stage; LLS: Late lactation stage

a, b Means with unlike letters in columns differ significantly $(\mathrm{P}<0.05)$.

a, b Aynı sütunda farklı harf taşıyan ortalamalar arası farklılıklar önemlidir $(\mathrm{P}<0.05)$

-: $\mathrm{P}>0.05 ; *: \mathrm{P}<0,05 ; * * *: \mathrm{P}<0.001$

\#: Means difference of pasture based from concentrate-based feeding systems; ${ }^{\$}$ : Means difference of $4 \leq$ ages goats from $4>$ ages goats;

\&: Means difference of single from twin birth types 
Table 3 presents the means of milk chemical composition and some milk quality parameters. Generally, although both milk chemical composition and some milk quality parameters were not affected from fixed factors (feeding systems, age and birth types), significant differences were detected among lactation $(\mathrm{P}<0.01$;
$\mathrm{P}<0.001$ ). While $\mathrm{L}^{*}$ values didn't display significant differences for fixed factors and lactation stages, $a^{*}$ and $b^{*}$ values were significantly different both in ELS for feeding systems $(\mathrm{P}<0.05 ; \mathrm{P}<0.01)$ and among lactation stages $(\mathrm{P}<0.01 ; \mathrm{P}<0.001)$ (Table 4).

Table 3. Pooled Means \pm SE of milk chemical composition and some milk quality traits for feeding systems, age and birth type

Tablo 3. Besleme sistemleri, yaş ve doğum tipi için sütün kimyasal kompozisyonu ve bazı süt kalite özelliklerine ait ortalamalar ve standart hatalar

\begin{tabular}{|c|c|c|c|c|c|}
\hline \multirow{2}{*}{\multicolumn{2}{|c|}{ Characters }} & \multirow[b]{2}{*}{ Pooled Means \pm SE } & \multicolumn{3}{|c|}{$\mathbf{P}$} \\
\hline & & & $\begin{array}{l}\text { Feeding systems } \\
\text { (Means difference }^{\#} \text { ) }\end{array}$ & $\begin{array}{c}\text { Age } \\
\left.\text { (Means difference }^{\$}\right)\end{array}$ & $\begin{array}{c}\text { Birth type } \\
\left.\text { (Means difference }^{\&}\right)\end{array}$ \\
\hline \multirow{4}{*}{$\mathrm{pH}$} & ELS & $6.662 \pm 0.01^{\mathrm{a}}$ & $0.036^{-}$ & $-0.003^{-}$ & $-0.044^{*}$ \\
\hline & MLS & $6.669 \pm 0.01^{\mathrm{a}}$ & $0.001^{-}$ & $-0.032^{-}$ & $0.034^{-}$ \\
\hline & LLS & $6.696 \pm 0.01^{\mathrm{b}}$ & $0.010^{-}$ & $-0.005^{-}$ & $-0.024^{-}$ \\
\hline & $\mathrm{P}$ & $* *$ & & & \\
\hline \multirow{4}{*}{$\begin{array}{l}\text { Electric } \\
\text { resistance } \\
(\mathrm{mV})\end{array}$} & ELS & $17.008 \pm 0.576^{\mathrm{b}}$ & $2.217^{-}$ & $-1.696^{-}$ & $-0.336^{-}$ \\
\hline & MLS & $16.535 \pm 0.664^{b}$ & $-1.801^{-}$ & $-0.699^{-}$ & $0.414^{-}$ \\
\hline & LLS & $15.036 \pm 0.738^{\mathrm{a}}$ & $-0.399^{-}$ & $-0.239^{-}$ & $1.218^{-}$ \\
\hline & $P$ & $* *$ & & & \\
\hline \multirow{4}{*}{ Fat $(\%)$} & ELS & $3.258 \pm 0.07^{\mathrm{a}}$ & $-0.069^{-}$ & $-0.210^{-}$ & $-0.094^{-}$ \\
\hline & MLS & $3.652 \pm 0.09^{\mathrm{b}}$ & $-0.119^{-}$ & $0.149^{-}$ & $-0.138^{-}$ \\
\hline & LLS & $3.461 \pm 0.10^{\mathrm{ab}}$ & $-0.196^{-}$ & $0.251^{-}$ & $-0.048^{-}$ \\
\hline & $\mathrm{P}$ & $* * *$ & & & \\
\hline \multirow{4}{*}{ Protein $(\%)$} & ELS & $2.837 \pm 0.04^{\mathrm{a}}$ & $0.195^{*}$ & $0.092^{-}$ & $-0.023^{-}$ \\
\hline & MLS & $3.062 \pm 0.07^{\mathrm{b}}$ & $0.028^{-}$ & $0.188^{-}$ & $0.026^{-}$ \\
\hline & LLS & $3.296 \pm 0.08^{c}$ & $-0.163^{-}$ & $0.160^{-}$ & $-0.018^{-}$ \\
\hline & $\mathrm{P}$ & $* * *$ & & & \\
\hline \multirow{4}{*}{ Lactose (\%) } & ELS & $5.044 \pm 0.02^{\mathrm{c}}$ & $0.039^{-}$ & $0.034^{-}$ & $0.112^{*}$ \\
\hline & MLS & $4.813 \pm 0.07^{\mathrm{b}}$ & $0.011^{-}$ & $0.007^{-}$ & $0.165^{-}$ \\
\hline & LLS & $4.532 \pm 0.06^{\mathrm{a}}$ & $0.054^{-}$ & $0.034^{-}$ & $-0.067^{-}$ \\
\hline & $\mathrm{P}$ & $* * *$ & & & \\
\hline \multirow{4}{*}{$\begin{array}{l}\text { Dry matter } \\
(\%)\end{array}$} & ELS & $10.586 \pm 0.15^{\mathrm{a}}$ & $0.470^{-}$ & $0.284^{-}$ & $-0.154^{-}$ \\
\hline & MLS & $12.494 \pm 0.20^{\mathrm{b}}$ & $-0.161^{-}$ & $0.367^{-}$ & $0.062^{-}$ \\
\hline & LLS & $12.294 \pm 0.15^{\mathrm{b}}$ & $-0.362^{-}$ & $0.470^{-}$ & $-0.172^{-}$ \\
\hline & $\mathrm{P}$ & $* * *$ & & & \\
\hline \multirow{4}{*}{$\operatorname{SCC}\left(x 10^{3}\right)$} & ELS & $599.600 \pm 76.51^{\mathrm{a}}$ & $65.449^{-}$ & $-226.576^{-}$ & $-258.500^{-}$ \\
\hline & MLS & $727.195 \pm 61.48^{\mathrm{a}}$ & $57.545^{-}$ & $-174.313^{-}$ & $-21.463^{-}$ \\
\hline & LLS & $1098.000 \pm 81.73^{\mathrm{b}}$ & $-36.892^{-}$ & $101.348^{-}$ & $113.827^{-}$ \\
\hline & $\mathrm{P}$ & $* * *$ & & & \\
\hline \multirow{4}{*}{$\begin{array}{c}\text { MDA } \\
(\mathrm{nmol} / \mathrm{ml})\end{array}$} & ELS & $8.406 \pm 0.49^{\mathrm{c}}$ & $2.808^{* *}$ & $-0.086^{-}$ & $1.018^{-}$ \\
\hline & MLS & $5.145 \pm 0.32^{\mathrm{b}}$ & $0.553^{-}$ & $-0.917^{-}$ & $0.037^{-}$ \\
\hline & LLS & $4.157 \pm 0.31^{\mathrm{a}}$ & $-0.716^{-}$ & $0.051^{-}$ & $0.513^{-}$ \\
\hline & $P$ & $* * *$ & & & \\
\hline
\end{tabular}

a, b, c Means with unlike letters in columns differ significantly $(\mathrm{P}<0.05)$.

a, b, c Aynı sütunda farklı harf taşıyan ortalamalar arası farklılıklar önemlidir $(\mathrm{P}<0.05)$

-: $\mathrm{P}>0.05 ; *: \mathrm{P}<0,05 ; * *: \mathrm{P}<0,01 ; * * *: \mathrm{P}<0.001$

\#: Means difference of pasture based from concentrate-based feeding systems; ${ }^{\$}$ : Means difference of $4 \leq$ ages goats from $4>$ ages goats;

\&: Means difference of single from twin birth types 
122 Akın Yakan - Hüseyin Özkan - Aysel Eraslan Şakar - Cafer Tayyar Ateş - Necmettin Ünal - Ömür Koçak - Gökhan Doğruer Ceyhan Özbeyaz

Table 4. Pooled Means \pm SE of milk color for feeding systems, age and birth type

Tablo 4. Besleme sistemleri, yaş ve doğum tipi için süt rengine ait ortalama ve standart hatalar

\begin{tabular}{|c|c|c|c|c|c|}
\hline \multirow{2}{*}{\multicolumn{2}{|c|}{ Characters }} & \multirow[b]{2}{*}{ Pooled Means \pm SE } & \multicolumn{3}{|c|}{$\mathbf{P}$} \\
\hline & & & $\begin{array}{c}\text { Feeding systems } \\
\text { (Means difference }^{\#} \text { ) }\end{array}$ & $\begin{array}{c}\text { Age } \\
\left.\text { (Means difference }^{\$}\right)\end{array}$ & $\begin{array}{c}\text { Birth type } \\
\text { (Means difference }^{\&} \text { ) }\end{array}$ \\
\hline \multirow{4}{*}{$\mathrm{L}^{*}$} & ELS & $91.584 \pm 0.15$ & $0.310^{-}$ & $0.249^{-}$ & $-0.543^{-}$ \\
\hline & MLS & $91.304 \pm 0.19$ & $0.140^{-}$ & $0.131^{-}$ & $-0.707^{-}$ \\
\hline & LLS & $91.386 \pm 0.15$ & $0.217^{-}$ & $0.261^{-}$ & $-0.525^{-}$ \\
\hline & $\mathrm{P}$ & - & & & \\
\hline \multirow{4}{*}{$a^{*}$} & ELS & $-3.385 \pm 0.07^{b}$ & $-0.308^{*}$ & $0.013^{-}$ & $-0.253^{-}$ \\
\hline & MLS & $-3.047 \pm 0.08^{\mathrm{a}}$ & $-0.114^{-}$ & $0.183^{-}$ & $-0.325^{-}$ \\
\hline & LLS & $-3.024 \pm 0.06^{\mathrm{a}}$ & $0.161^{-}$ & $0.127^{-}$ & $-0.200^{-}$ \\
\hline & $\mathrm{P}$ & $* * *$ & & & \\
\hline \multirow{4}{*}{$b^{*}$} & ELS & $5.499 \pm 0.22^{\mathrm{a}}$ & $1.175^{* *}$ & $-0.028^{-}$ & $-0.130^{-}$ \\
\hline & MLS & $5.915 \pm 0.22^{\mathrm{b}}$ & $0.555^{-}$ & $-0.123^{-}$ & $0.068^{-}$ \\
\hline & LLS & $6.130 \pm 0.21^{\mathrm{b}}$ & $-0.168^{-}$ & $0.109^{-}$ & $0.441^{-}$ \\
\hline & $\mathrm{P}$ & $*$ & & & \\
\hline
\end{tabular}

a, b Means with unlike letters in columns differ significantly $(\mathrm{P}<0.05)$.

a, b Aynı sütunda farklı harf taşıyan ortalamalar arası farklılıklar önemlidir $(\mathrm{P}<0.05)$

-: $\mathrm{P}>0.05 ; *: \mathrm{P}<0,05 ; * *: \mathrm{P}<0,01 ; * * *: \mathrm{P}<0.001$

\#: Means difference of pasture based from concentrate-based feeding systems; ${ }^{\$}$ : Means difference of $4 \leq$ ages goats from $4>$ ages goats;

\&: Means difference of single from twin birth types

Table 5. Pooled Means \pm SE of milk mineral matters for feeding systems, age and birth type

Tablo 5. Besleme sistemleri, yaş ve doğum tipi için sütteki mineral maddelere ait ortalamalar ve standart hatalar

\begin{tabular}{|c|c|c|c|c|c|}
\hline \multirow{2}{*}{\multicolumn{2}{|c|}{ Characters }} & \multirow[b]{2}{*}{ Pooled Means \pm SE } & \multicolumn{3}{|c|}{$\mathbf{P}$} \\
\hline & & & $\begin{array}{c}\text { Feeding systems } \\
\left(\text { Means difference }^{\#}\right)\end{array}$ & $\begin{array}{c}\text { Age } \\
\left.\text { (Means difference }{ }^{\$}\right)\end{array}$ & $\begin{array}{c}\text { Birth type } \\
\left.\text { (Means difference }^{\&}\right)\end{array}$ \\
\hline \multirow{4}{*}{$\begin{array}{c}\mathrm{Ca} \\
(\mathrm{mg} / \mathrm{ml})\end{array}$} & ELS & $888.696 \pm 30.33^{c}$ & $135.333^{*}$ & $52.522^{-}$ & $-67.188^{-}$ \\
\hline & MLS & $590.380 \pm 18.23^{\mathrm{a}}$ & $71.500^{-}$ & $17.348^{-}$ & $-46.348^{-}$ \\
\hline & LLS & $664.565 \pm 21.75^{b}$ & $34.000^{-}$ & $44.174^{-}$ & $-20.174^{-}$ \\
\hline & $\mathrm{P}$ & $* * *$ & & & \\
\hline \multirow{4}{*}{$\begin{array}{c}\mathrm{Mg} \\
(\mathrm{ng} / \mathrm{ml})\end{array}$} & ELS & $94.194 \pm 3.57^{b}$ & $8.217^{-}$ & $3.372^{-}$ & $-3.439^{-}$ \\
\hline & MLS & $68.957 \pm 2.45^{\mathrm{a}}$ & $8.800^{-}$ & $1.717^{-}$ & $-0.217^{-}$ \\
\hline & LLS & $73.125 \pm 3.05^{\mathrm{a}}$ & $1.450^{-}$ & $3.400^{-}$ & $0.400^{-}$ \\
\hline & $\mathrm{P}$ & $* * *$ & & & \\
\hline \multirow{4}{*}{$\mathrm{Fe}(\mathrm{ng} / \mathrm{ml})$} & ELS & $2.441 \pm 0.35^{\mathrm{c}}$ & $-0.217^{-}$ & $0.557^{-}$ & $-0.563^{-}$ \\
\hline & MLS & $1.447 \pm 0.19^{\mathrm{b}}$ & $-0.820^{*}$ & $0.281^{-}$ & $-0.001^{-}$ \\
\hline & LLS & $0.907 \pm 0.04^{\mathrm{a}}$ & $0.110^{-}$ & $0.187^{*}$ & $0.103^{-}$ \\
\hline & $\mathrm{P}$ & $* * *$ & & & \\
\hline \multirow{4}{*}{$\begin{array}{c}\mathrm{Zn} \\
(\mathrm{ng} / \mathrm{ml})\end{array}$} & ELS & $4.315 \pm 0.34^{\mathrm{b}}$ & $0.142^{-}$ & $0.502^{-}$ & $0.778^{-}$ \\
\hline & MLS & $2.690 \pm 0.08^{\mathrm{a}}$ & $0.055^{-}$ & $0.429^{*}$ & $0.351^{*}$ \\
\hline & LLS & $2.868 \pm 0.158^{\mathrm{a}}$ & $-0.205^{-}$ & $0.618^{-}$ & $0.422^{-}$ \\
\hline & $\mathrm{P}$ & $* * *$ & & & \\
\hline \multirow{4}{*}{$\begin{array}{c}\mathrm{Cu} \\
(\mathrm{ng} / \mathrm{ml})\end{array}$} & ELS & $0.410 \pm 0.03^{\mathrm{c}}$ & $-0.018^{-}$ & $-0.062^{-}$ & $-0.038^{-}$ \\
\hline & MLS & $0.248 \pm 0.01^{\mathrm{b}}$ & $-0.015^{-}$ & $-0.034^{-}$ & $-0.026^{-}$ \\
\hline & LLS & $0.159 \pm 0.01^{\mathrm{a}}$ & $0.020^{-}$ & $-0.003^{-}$ & $-0.017^{-}$ \\
\hline & $\mathrm{P}$ & $* * *$ & & & \\
\hline
\end{tabular}

a, b, c Means with unlike letters in columns differ significantly $(\mathrm{P}<0.05)$.

a, b, c Aynı sütunda farklı harf taşıyan ortalamalar arası farklılıklar önemlidir $(\mathrm{P}<0.05)$

-: $\mathrm{P}>0.05 ; *: \mathrm{P}<0,05 ; * *: \mathrm{P}<0,01 ; * * *: \mathrm{P}<0.001$

\#: Means difference of pasture based from concentrate-based feeding systems; $\$$ : Means difference of $4 \leq$ ages goats from $4>$ ages goats;

\&: Means difference of single from twin birth types 
All minerals significantly decreased during lactation $(\mathrm{P}<0.05 ; \mathrm{P}<0.01 ; \mathrm{P}<0.001)$ and $\mathrm{Ca}$ levels were higher in PBFG compared to CBFG at ELS $(\mathrm{P}<0.05)$. Also, Fe levels in LLS and Zn levels in MLS were higher $4 \leq$ ages than $4>$ ages goats $(\mathrm{P}<0.05)$ (Table 5).

Values for fatty acid composition of milk cream are given in Table 6 . The main fatty acids were palmitic acid (C16:0) and oleic acid (C18:1) followed by stearic acid (C18:0) in all experimental groups. All fatty acids with a few exceptions were affected by lactation stages $(\mathrm{P}<0.05$; $\mathrm{P}<0.01 ; \mathrm{P}<0.001)$ and on the other hand, short chain fatty acid (eight carbons or shorter) levels were different except butyric acid in LLS for feeding system effect $(\mathrm{P}<0.05$, $\mathrm{P}<0.001)$. The best part of medium chain fatty acids (between fourteen and eighteen carbons) was not different for feeding systems but some of them were different. Table 7 presents the sums and ratios and index values obtained from fatty acids. Significant differences in the all total percentage of fatty acids (Saturated Fatty Acids (SFA), Monounsaturated Fatty Acid (MUFA), Polyunsaturated Fatty Acid (PUFA) etc.) were detected in lactation stages $(\mathrm{P}<0.05 ; \quad \mathrm{P}<0.001)$, but same differentiation in the all total percentages of fatty acids was not detected for feeding systems. The all sum and ratio based on milk fatty acids were not statistically different for ages $(\mathrm{P}>0.05)$. The odour index $(\mathrm{OI})$ was different between lactation stages $(\mathrm{P}<0.001)$ and MLS and LLS were different for feeding systems $(\mathrm{P}<0.05$; $\mathrm{P}<0.001)$ but not affected from ages and birth types.

Table 6. Pooled Means \pm SE of milk fatty acid composition for feeding systems, age and birth type

Tablo 6. Besleme sistemleri, yaş ve doğum tipi için süt yağ asidi kompoziyonuna ait ortalamalar ve standart hatalar

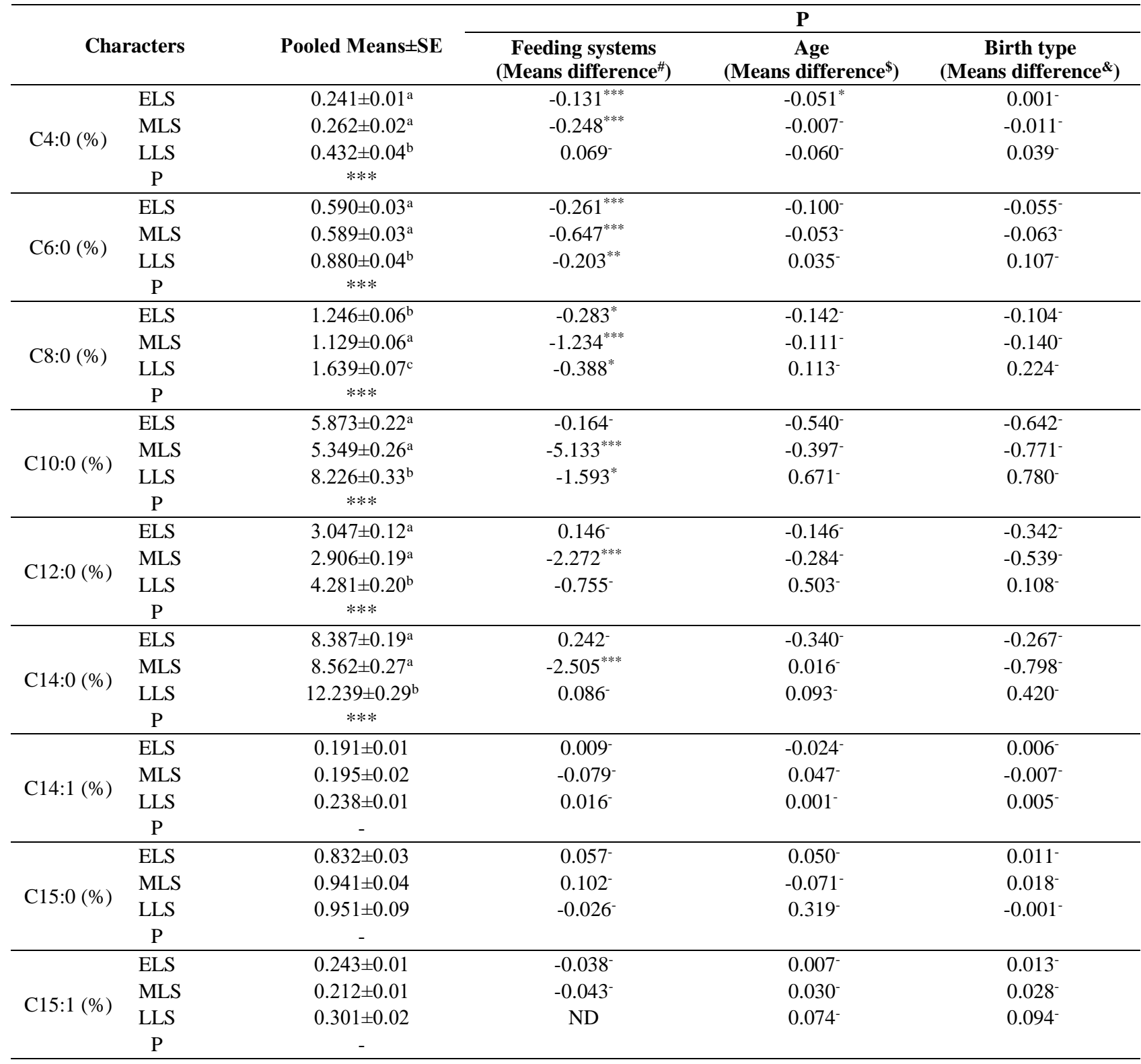


124 Akın Yakan - Hüseyin Özkan - Aysel Eraslan Şakar - Cafer Tayyar Ateş - Necmettin Ünal - Ömür Koçak - Gökhan Doğruer -

Ceyhan Özbeyaz

Table 6. Pooled Means \pm SE of milk fatty acid composition for feeding systems, age and birth type (continued)

Tablo 6. Besleme sistemleri, yaş ve doğum tipi için süt yağ asidi kompoziyonuna ait ortalamalar ve standart hatalar (devam)

\begin{tabular}{|c|c|c|c|c|c|}
\hline \multirow{2}{*}{\multicolumn{2}{|c|}{ Characters }} & \multirow[b]{2}{*}{ Pooled Means \pm SE } & \multicolumn{3}{|c|}{$\mathbf{P}$} \\
\hline & & & $\begin{array}{l}\text { Feeding systems } \\
\left.\text { (Means difference }{ }^{\#}\right)\end{array}$ & $\begin{array}{c}\text { Age } \\
\left.\text { (Means difference }^{\$}\right) \\
\end{array}$ & $\begin{array}{c}\text { Birth type } \\
\left.\text { (Means difference }^{\&}\right)\end{array}$ \\
\hline \multirow{4}{*}{$\mathrm{C} 16: 0(\%)$} & ELS & $26.939 \pm 0.46^{\mathrm{a}}$ & $-0.277^{-}$ & $0.311^{-}$ & $-0.159^{-}$ \\
\hline & MLS & $30.225 \pm 0.96^{\mathrm{b}}$ & $-0.982^{-}$ & $0.680^{-}$ & $-0.590^{-}$ \\
\hline & LLS & $37.408 \pm 1.31^{\mathrm{c}}$ & $2.203^{-}$ & $-4.372^{-}$ & $-2.777^{-}$ \\
\hline & $\mathrm{P}$ & $* * *$ & & & \\
\hline \multirow{4}{*}{ C16:1 (\%) } & ELS & $0.693 \pm 0.03^{\mathrm{a}}$ & $-0.071^{-}$ & $0.050^{-}$ & $0.001^{-}$ \\
\hline & MLS & $0.727 \pm 0.02^{\mathrm{a}}$ & $0.021^{-}$ & $-0.064^{-}$ & $-0.020^{-}$ \\
\hline & LLS & $0.878 \pm 0.06^{\mathrm{b}}$ & $-0.175^{-}$ & $-0.002^{-}$ & $-0.045^{-}$ \\
\hline & $\mathrm{P}$ & $*$ & & & \\
\hline \multirow{4}{*}{$\mathrm{C} 17: 0(\%)$} & ELS & $0.845 \pm 0.02^{\mathrm{b}}$ & $0.014^{-}$ & $0.077^{-}$ & $-0.031^{-}$ \\
\hline & MLS & $0.758 \pm 0.02^{\mathrm{a}}$ & $0.249^{* * *}$ & $0.014^{-}$ & $-0.003^{-}$ \\
\hline & LLS & $0.730 \pm 0.04^{\mathrm{a}}$ & $0.145^{*}$ & $0.060^{-}$ & $-0.021^{-}$ \\
\hline & $\mathrm{P}$ & $* *$ & & & \\
\hline \multirow{4}{*}{ C17:1 (\%) } & ELS & $0.243 \pm 0.01$ & $-0.091^{* * *}$ & $0.039^{-}$ & $0.024^{-}$ \\
\hline & MLS & $0.218 \pm 0.02$ & $-0.043^{-}$ & $0.060^{-}$ & $0.003^{-}$ \\
\hline & LLS & $0.256 \pm 0.02$ & $0.051^{-}$ & $-0.010^{-}$ & $-0.016^{-}$ \\
\hline & $\mathrm{P}$ & - & & & \\
\hline \multirow{4}{*}{ C18:0 (\%) } & ELS & $16.908 \pm 0.50^{\mathrm{b}}$ & $1.349^{-}$ & $0.129^{-}$ & $0.443^{-}$ \\
\hline & MLS & $16.350 \pm 0.81^{\mathrm{b}}$ & $5.304^{* * *}$ & $0.108^{-}$ & $0.508^{-}$ \\
\hline & LLS & $8.290 \pm 0.56^{\mathrm{a}}$ & $1.465^{-}$ & $0.983^{-}$ & $0.445^{-}$ \\
\hline & $\mathrm{P}$ & $* * *$ & & & \\
\hline \multirow{4}{*}{ C18:1 (\%) } & ELS & $27.227 \pm 0.531^{b}$ & $-1.167^{-}$ & $0.602^{-}$ & $1.360^{-}$ \\
\hline & MLS & $25.388 \pm 0.76^{\mathrm{b}}$ & $6.443^{* * * *}$ & $0.450^{-}$ & $2.333^{-}$ \\
\hline & LLS & $20.016 \pm 0.68^{a}$ & $-0.614^{-}$ & $1.208^{-}$ & $0.490^{-}$ \\
\hline & $\mathrm{P}$ & $* * *$ & & & \\
\hline \multirow{4}{*}{$\begin{array}{l}\text { C18:2 n6 } \\
\quad(\%)\end{array}$} & ELS & $3.730 \pm 0.10^{c}$ & $0.135^{-}$ & $-0.321^{-}$ & $-0.612^{* *}$ \\
\hline & MLS & $3.117 \pm 0.12^{\mathrm{b}}$ & $-0.255^{-}$ & $-0.202^{-}$ & $0.032^{-}$ \\
\hline & LLS & $2.162 \pm 0.06^{\mathrm{a}}$ & $-0.508^{* * *}$ & $0.436^{* * *}$ & $0.233^{-}$ \\
\hline & $\mathrm{P}$ & $* * *$ & & & \\
\hline \multirow{4}{*}{$\begin{array}{l}\text { C18:3 n6 } \\
(\%)\end{array}$} & ELS & $1.017 \pm 0.09^{\mathrm{b}}$ & $0.566^{* *}$ & $-0.131^{-}$ & $-0.359^{-}$ \\
\hline & MLS & $1.194 \pm 0.12^{\mathrm{b}}$ & $0.006^{-}$ & $-0.204^{-}$ & $-0.160^{-}$ \\
\hline & LLS & $0.631 \pm 0.08^{\mathrm{a}}$ & $0.125^{-}$ & $-0.092^{-}$ & $-0.010^{-}$ \\
\hline & $\mathrm{P}$ & $*$ & & & \\
\hline \multirow{4}{*}{$\begin{array}{c}\mathrm{C} 18: 3 \mathrm{n} 3 \\
(\%)\end{array}$} & ELS & $0.863 \pm 0.06^{\mathrm{b}}$ & $0.270^{*}$ & $0.081^{-}$ & $0.124^{-}$ \\
\hline & MLS & $0.824 \pm 0.06^{\mathrm{b}}$ & $0.138^{-}$ & $-0.014^{-}$ & $0.060^{-}$ \\
\hline & LLS & $0.488 \pm 0.04^{\mathrm{a}}$ & $-0.042^{-}$ & $0.052^{-}$ & $0.087^{-}$ \\
\hline & $\mathrm{P}$ & $* * *$ & & & \\
\hline \multirow{4}{*}{$\mathrm{C} 20: 0(\%)$} & ELS & $0.333 \pm 0.02^{\mathrm{a}}$ & $0.005^{-}$ & $-0.037^{-}$ & $-0.032^{-}$ \\
\hline & MLS & $0.728 \pm 0.04^{\mathrm{b}}$ & $0.682^{* * * *}$ & $-0.195^{*}$ & $0.033^{-}$ \\
\hline & LLS & $0.350 \pm 0.02^{\mathrm{a}}$ & $0.117^{* *}$ & $-0.015^{-}$ & $-0.022^{-}$ \\
\hline & $\mathrm{P}$ & $* * *$ & & & \\
\hline \multirow{4}{*}{$\begin{array}{c}\mathrm{C} 20: 4 \mathrm{n} 6 \\
(\%)\end{array}$} & ELS & $0.167 \pm 0.01^{\mathrm{a}}$ & $-0.026^{*}$ & $-0.008^{-}$ & $-0.019^{-}$ \\
\hline & MLS & $0.186 \pm 0.02^{\mathrm{a}}$ & $-0.004^{-}$ & $-0.016^{-}$ & $-0.063^{*}$ \\
\hline & LLS & $0.247 \pm 0.01^{\mathrm{b}}$ & ND & $0.040^{-}$ & $0.038^{-}$ \\
\hline & $\mathrm{P}$ & * & & & \\
\hline \multirow{4}{*}{$\mathrm{C} 22: 0(\%)$} & ELS & $0.113 \pm 0.01$ & $0.024^{-}$ & $0.007^{-}$ & $-0.011^{-}$ \\
\hline & MLS & $0.230 \pm 0.03$ & $0.264^{* * * *}$ & $-0.056^{-}$ & $0.012^{-}$ \\
\hline & LLS & ND & ND & ND & ND \\
\hline & $\mathrm{P}$ & - & & & \\
\hline \multirow{4}{*}{$\mathrm{C} 24: 0(\%)$} & ELS & $0.200 \pm 0.01$ & $0.031^{*}$ & $-0.009^{-}$ & $-0.005^{-}$ \\
\hline & MLS & $0.222 \pm 0.02$ & $0.162^{\text {** }}$ & $-0.029^{-}$ & $-0.016^{-}$ \\
\hline & LLS & ND & ND & ND & ND \\
\hline & $\mathrm{P}$ & - & & & \\
\hline \multicolumn{6}{|c|}{$\begin{array}{l}\text { a, b, c Means with unlike letters in columns differ significantly }(\mathrm{P}<0.05) \text {. } \\
\text { a, b, c Aynı sütunda farklı harf taș1yan ortalamalar arası farklılıklar önemlidir }(\mathrm{P}<0.05)\end{array}$} \\
\hline
\end{tabular}


Table 7. Pooled Means \pm SE of sum and ratio based on milk fatty acids for feeding systems, age and birth type Tablo 7. Besleme sistemleri, yaş ve doğum tipi için süt yağ asidi oranlarına ait ortalamalar ve standart hatalar

\begin{tabular}{|c|c|c|c|c|c|}
\hline \multirow{2}{*}{\multicolumn{2}{|c|}{ Characters }} & \multirow[b]{2}{*}{ Pooled Means \pm SE } & \multicolumn{3}{|c|}{$\mathbf{P}$} \\
\hline & & & $\begin{array}{c}\text { Feeding systems } \\
\left.\text { (Means difference }^{\#}\right)\end{array}$ & $\begin{array}{c}\text { Age } \\
\text { (Means difference }^{\$} \text { ) }\end{array}$ & $\begin{array}{c}\text { Birth type } \\
\left.\text { (Means difference }^{\&}\right) \\
\end{array}$ \\
\hline \multirow{4}{*}{ SFA $(\%)$} & ELS & $66.675 \pm 0.62^{\mathrm{a}}$ & $0.379^{-}$ & $-0.329^{-}$ & $-0.566^{-}$ \\
\hline & MLS & $68.909 \pm 0.89^{\mathrm{b}}$ & $-7.471^{* * *}$ & $0.723^{-}$ & $0.358^{-}$ \\
\hline & LLS & $75.997 \pm 0.97^{\mathrm{c}}$ & $0.532^{-}$ & $-1.339^{-}$ & $0.389^{-}$ \\
\hline & $\mathrm{P}$ & $* * *$ & & & \\
\hline \multirow{4}{*}{ MUFA (\%) } & ELS & $28.561 \pm 0.55^{\mathrm{b}}$ & $-1.347^{-}$ & $0.689^{-}$ & $1.418^{-}$ \\
\hline & MLS & $26.332 \pm 1.00^{\mathrm{b}}$ & $5.997^{* *}$ & $0.863^{-}$ & $-0.450^{-}$ \\
\hline & LLS & $20.841 \pm 0.86^{\mathrm{a}}$ & $-0.419^{-}$ & $1.118^{-}$ & $-0.339^{-}$ \\
\hline & $\mathrm{P}$ & $* * *$ & & & \\
\hline \multirow{4}{*}{ PUFA (\%) } & ELS & $5.764 \pm 0.19^{c}$ & $0.968^{*}$ & $-0.360^{-}$ & $-0.852^{*}$ \\
\hline & MLS & $4.711 \pm 0.14^{\mathrm{b}}$ & $0.970^{* *}$ & $-0.069^{-}$ & $-0.190^{-}$ \\
\hline & LLS & $3.159 \pm 0.15^{\mathrm{a}}$ & $-0.112^{-}$ & $0.213^{-}$ & $-0.042^{-}$ \\
\hline & $\mathrm{P}$ & $* * *$ & & & \\
\hline \multirow{4}{*}{ UFA (\%) } & ELS & $34.325 \pm 0.62^{\mathrm{c}}$ & $-0.379^{-}$ & $0.329^{-}$ & $0.566^{-}$ \\
\hline & MLS & $31.121 \pm 1.05^{\mathrm{b}}$ & $6.829^{* *}$ & $0.624^{-}$ & $-0.754^{-}$ \\
\hline & LLS & $24.001 \pm 0.97^{\mathrm{a}}$ & $-0.532^{-}$ & $1.332^{-}$ & $-0.381^{-}$ \\
\hline & $\mathrm{P}$ & $* * *$ & & & \\
\hline \multirow{4}{*}{$\begin{array}{c}\text { PUFA/SFA } \\
(\%)\end{array}$} & ELS & $0.088 \pm 0.01^{\mathrm{c}}$ & $0.014^{*}$ & $-0.004^{-}$ & $-0.012^{-}$ \\
\hline & MLS & $0.068 \pm 0.01^{\mathrm{b}}$ & $0.023^{* *}$ & $0.002^{-}$ & $0.007^{-}$ \\
\hline & LLS & $0.042 \pm 0.01^{\mathrm{a}}$ & $-0.002^{-}$ & $0.003^{-}$ & $-0.001^{-}$ \\
\hline & $\mathrm{P}$ & $* * *$ & & & \\
\hline \multirow{4}{*}{$\begin{array}{c}\text { UFA/SFA } \\
(\%)\end{array}$} & ELS & $0.529 \pm 0.02^{\mathrm{b}}$ & $-0.015^{-}$ & $0.006^{-}$ & $0.009^{-}$ \\
\hline & MLS & $0.452 \pm 0.03^{\mathrm{b}}$ & $0.152^{*}$ & $0.051^{-}$ & $-0.006^{-}$ \\
\hline & LLS & $0.321 \pm 0.02^{\mathrm{a}}$ & $-0.013^{-}$ & $0.020^{-}$ & $-0.011^{-}$ \\
\hline & $\mathrm{P}$ & $* * *$ & & & \\
\hline \multirow{4}{*}{ n6 } & ELS & $4.909 \pm 0.16^{\mathrm{c}}$ & $0.684^{*}$ & $-0.460^{-}$ & $-0.990^{* *}$ \\
\hline & MLS & $3.955 \pm 0.12^{\mathrm{b}}$ & $0.714^{* *}$ & $0.026^{-}$ & $-0.119^{-}$ \\
\hline & LLS & $2.721 \pm 0.12^{\mathrm{a}}$ & $-0.143^{-}$ & $0.234^{-}$ & $-0.039^{-}$ \\
\hline & $\mathrm{P}$ & $* * *$ & & & \\
\hline \multirow{4}{*}{ n3 } & ELS & $0.863 \pm 0.06^{\mathrm{b}}$ & $0.269^{*}$ & $0.082^{-}$ & $0.125^{-}$ \\
\hline & MLS & $0.754 \pm 0.06^{\mathrm{b}}$ & $0.238^{*}$ & $0.028^{-}$ & $-0.020^{-}$ \\
\hline & LLS & $0.506 \pm 0.03^{\mathrm{a}}$ & $-0.095^{-}$ & $0.047^{-}$ & $0.094^{-}$ \\
\hline & $\mathrm{P}$ & $* * *$ & & & \\
\hline \multirow{4}{*}{$\mathrm{n} 6 / \mathrm{n} 3$} & ELS & $6.745 \pm 0.25^{\mathrm{b}}$ & $-0.644^{-}$ & $-0.925^{-}$ & $-1.646^{* *}$ \\
\hline & MLS & $6.010 \pm 0.40^{\mathrm{a}}$ & $-0.836^{-}$ & $-0.630^{-}$ & $0.390^{-}$ \\
\hline & LLS & $6.745 \pm 0.30^{\mathrm{b}}$ & $-0.882^{-}$ & $0.511^{-}$ & $-0.296^{-}$ \\
\hline & $\mathrm{P}$ & $*$ & & & \\
\hline \multirow{4}{*}{ NV } & ELS & $1.751 \pm 0.07^{\mathrm{c}}$ & $-0.136^{-}$ & $0.137^{-}$ & $0.247^{-}$ \\
\hline & MLS & $1.329 \pm 0.09^{\mathrm{b}}$ & $0.626^{* *}$ & $0.062^{-}$ & $0.048^{-}$ \\
\hline & LLS & $0.771 \pm 0.06^{\mathrm{a}}$ & $-0.024^{-}$ & $0.079^{-}$ & $-0.011^{-}$ \\
\hline & $\mathrm{P}$ & $* * *$ & & & \\
\hline \multirow{4}{*}{ AI } & ELS & $1.597 \pm 0.05^{\mathrm{a}}$ & $0.076^{-}$ & $-0.061^{-}$ & $-0.079^{-}$ \\
\hline & MLS & $1.927 \pm 0.09^{b}$ & $-0.907^{* * *}$ & $-0.152^{-}$ & $-0.128^{-}$ \\
\hline & LLS & $2.704 \pm 0.14^{\mathrm{c}}$ & $-0.089^{-}$ & $-0.063^{-}$ & $0.021^{-}$ \\
\hline & $\mathrm{P}$ & $* * *$ & & & \\
\hline \multirow{4}{*}{ TI } & ELS & $1.607 \pm 0.05^{\mathrm{a}}$ & $0.042^{-}$ & $-0.042^{-}$ & $-0.100^{-}$ \\
\hline & MLS & $2.101 \pm 0.10^{\mathrm{b}}$ & $-0.768^{* *}$ & $-0.188^{-}$ & $-0.072^{-}$ \\
\hline & LLS & $2.675 \pm 0.14^{\mathrm{c}}$ & $0.085^{-}$ & $-0.214^{-}$ & $-0.127^{-}$ \\
\hline & $\mathrm{P}$ & $* * *$ & & & \\
\hline \multirow{4}{*}{ OI } & ELS & $7.488 \pm 0.43^{\mathrm{a}}$ & $-0.731^{-}$ & $-0.643^{-}$ & $0.430^{-}$ \\
\hline & MLS & $6.461 \pm 0.36^{\mathrm{a}}$ & $-5.891^{* * *}$ & $0.115^{-}$ & $-0.571^{-}$ \\
\hline & LLS & $10.609 \pm 0.51^{\mathrm{b}}$ & $-2.566^{*}$ & $1.244^{-}$ & $1.376^{-}$ \\
\hline & $\mathrm{P}$ & $* * *$ & & & \\
\hline
\end{tabular}

a, b, c Means with unlike letters in columns differ significantly $(\mathrm{P}<0.05)$.

a, b, c Aynı sütunda farklı harf taşıyan ortalamalar arası farklılıklar önemlidir $(\mathrm{P}<0.05)$

-: $\mathrm{P}>0.05 ; *: \mathrm{P}<0,05 ; * *: \mathrm{P}<0,01 ; * * *: \mathrm{P}<0.001$

\#: Means difference of pasture based from concentrate-based feeding systems; ${ }^{\$}$ : Means difference of $4 \leq$ ages goats from $4>$ ages goats; \&: Means difference of single from twin birth types 


\section{Discussion and Conclusion}

Lactation Duration and Milk Yield: Longer lactation is generally desired because length of lactation is one of most important factors for lactation milk yield (14). This duration can change based on breed which is determined according to dairy, meat type or indigenous breeds. Dairy goats have a long lactation stage between $7-$ 10 months (2). In this research, lactation durations were 210.48 days and it was not affected from fixed factors (feeding systems, age and birth types), but lactation milk yield was $238.185 \mathrm{~kg}$ and it was higher in PBFG compared to $\mathrm{CBFG}$ (126 kg means difference) $(\mathrm{P}<0.001)$, higher in older goats than younger (42 $\mathrm{kg}$ means difference) $(\mathrm{P}<0.05)$. Classical knowledge says that birth types is important on milk yield, but this study result were not compatible with this classical knowledge because there was no difference between single and twin birth type for lactation milk yield. Both lactation length and lactation milk yield were lower compared to previous analysis on Damascus breed (17) because the experimental groups of this study were not selected as herd but reared for milk yield under local breeding conditions (16). But, detected lactation length and milk yield in this study proved that lactation was longer and milk yield was higher compared to other indigenous local goat breeds (11) or similar to Turkish Saanen goats (25). On the other hand, it is generally expected that lactation milk yield of goats with diets rich in concentrates may be higher than goats fed with pasture-based feeding systems due to higher energy intakes in concentrate-based feeding systems (23), but in this study findings were higher in PBFG compared to CBFG (126 kg means difference). This finding was interpreted that PBFG obtained sufficient energy levels from pasture grazing. Also, in terms of carcass and meat quality, Yakan et al. (45) stated that that higher production in intensive systems is not expected to be the same in extensive or semi-intensive production systems for goats because goats intrinsically need to graze more than other ruminant species (cow and sheep etc). When lactation stages are examined, although ELS and MLS showed similar milk yield in both experimental groups, LLS milk yields were lower than first two lactation stages $(\mathrm{P}<0.001)$. This decrease in milk yield in LLS might be caused by oestrus and due to pregnancy of goats in this period because it is well known that oestrogen and progesterone hormones are suppressor factors for lactation (4). The fact that the goats were detected to pregnancy from flock owner records.

Milk quality traits: $\mathrm{pH}$ is a very important parameter for determining milk quality because it converts milk to cheese via coagulation of proteins. $\mathrm{pH}$ values in this study were compatible with the findings of Sampelayo et al. (34) for Granada goats fed different concentrate feed. Although $\mathrm{pH}$ values were similar at all lactation stages for feeding systems, ages and birth types (except ELS), there were significant differences among lactation stages $(\mathrm{P}<0.01)$. Other important effects were not observed for feeding systems on the electric resistance, fat, protein (except ELS), lactose, dry matter, SCC and MDA (except ELS) and milk composition (fat, protein, lactose and dry matter) was generally consistent with numerous authors (3, 30, 31) but, all the above parameters were significantly affected from lactation stages $(\mathrm{P}<0.01 ; \mathrm{P}<0.001)$. Milk fat and dry matter levels increased with lactation stage as consistent with lactation physiology and lactose levels decreased. Similar findings were indicated for Beetal and their crossbreed of goats by Prasad et al. (30). It is thought that the reason for the increase in milk fat ratio was due to both decreases in milk yield as a result of lactation and increases in roughage intake in LLS. Intake of cellulose volume is the reason of high milk fat ratio (10) and LLS was lengthened due to drying grass during the study period.

SCC forms the basis of abnormal milk control programs and the legal limit in USA was established by Food and Drug Administration for goats is $1000 \times 10^{3} / \mathrm{ml}$ (26). Although results from the data in this study indicate that SCC was increased with stage of lactation, the highest SCC was observed in LLS, the values were just at the legal limit. Cell counts for uninfected mammary glands have been shown to increase with period of lactation (19). While SCC in this study was not affected by fixed factors, it was found to be significantly different among lactation stages $(\mathrm{P}<0.001)$. Detected SCC in this work was generally compatible with findings of Orman et al. (25) and Gomes et al. (15). According to these SCC results, it can be argued that SCC increased as lactation went by, regardless of the presence of mastitis. Also, Hinckley and Williams (18) stated that no significant correlation was revealed between SCC and the leucocyte count. On the other hand, while SCC increased with lactation periods, milk yields decreased. This means that as SCC increases, milk yield decreases in goats. This finding is consistent with the findings of Raynal-Ljutovac et al. (32) and Orman et al. (25). Electric resistance of milk depends on the cations and anions levels in milk (6). While electric resistance was not affected from fixed factors, it significantly decreased with lactation stages $(\mathrm{P}<0.01)$. That finding is compatible with the study by Das and Singh (6).

Milk MDA level is the most generally preferred indicator to evaluate peroxidation status (24). MDA levels were significantly higher in PBFG in ELS compared to CBFG $(\mathrm{P}<0.01)$. Experimental goats were in shelter during the last 3 months of pregnancy period. After PBFG goats gave birth, they directly went to pasture during daytime and probably walked about $8-10 \mathrm{~km} /$ day in pasture. This walking effect could be the reason to 
oxidative stress and that MDA levels might be higher in PBFG compared to CBFG. Later, this difference in MDA levels disappeared in mid and LLS since goats might have been accustomed to pasture. On the other hand, MDA levels decreased in time by lactation while SCC increased by lactation. Some authors $(40,47)$ reported that MDA and SCC were related to mastitis in cows. This result was not confirmed by our findings. On the contrary, it is shown that MDA level is not indicator of mastitis in goats, similar to SCC. Some authors $(40,47)$ investigated milk MDA levels and mastitis relationship but no study found MDA levels in all lactation stages and in different feeding systems in goats.

The color of butterfat contributes to the color of milk just like other milk components such as proteins and volatile compound. Reflectance spectra for whole milk showed that there was no effect in $\mathrm{L}^{*}$ for both fixed factors and lactation stages. $b^{*}$ index was significantly differ for feeding system in ELS, but this effect disappeared in MLS and LLS. In the study area, while the pasture was green in ELS, it dried in MLS and LLS. Higher $b^{*}$ values presumably depended on consumed green grass which contained flavonoids. Solah et al., (38) reported that $b^{*}$ values are affected by cows' feeding systems such as pasture, silage and grain feed etc. It is shown that similar effect is valid for goats. $a^{*}$ index had negative values for all groups and stages and this finding is compatible with the results of Rufian-Henares et al., (33) but PBFG came close to zero $(\mathrm{P}<0.001)$.

The concentration of milk macro-minerals does not alter much, but they depend on the breed, diet, lactation stage and mammary health of goats. Important changes in contents of the macro-minerals in goat milk were detected during lactation (27). Total calcium is the most abundant mineral in milk (32) and was affected by ELS $(\mathrm{P}<0.05)$ and lactation stages $(\mathrm{P}<0.001)$. Calcium values were lower for different goat breeds $(3,29,31)$. The reason of this difference was probably caused by breed effect. Other minerals in milk, $\mathrm{Mg}, \mathrm{Fe}, \mathrm{Zn}$ and $\mathrm{Cu}$, also significantly decreased during lactation $(\mathrm{P}<0.001)$. This change of milk mineral concentrations is consistent with some studies ( 1 , 28).

Feed is a very important parameter in milk fatty acid composition (29). Twenty-two fatty acids were detected from butyric acid (C4:0) to lignoceric acid (C24:0) for both feeding systems. While most of them, particularly short-chain fatty acids (from $\mathrm{C} 4: 0$ to $\mathrm{C} 10: 0$ ), were significantly different for feeding systems, there were some exceptions for lactation stages $(\mathrm{P}<0.05$; $\mathrm{P}<0.01$; $\mathrm{P}<0.001)$. Kondyli and Katsiari (20) stated that variations in milk fatty acids were generally caused by seasonal variations and appeared to be diet-related. The three most important fatty acids in quantitative terms, palmitic (C16:0), stearic (C18:0) and oleic acid (C18:1) accounted for approximately $>75 \%$. This ratio was consistent with the findings of some authors who studied Damascus goats (17) and other goat breeds $(9,41,42)$. These major milk fatty acids may be affected by stage of lactation $(9,41)$. Similar to this finding, while palmitic acid increased $(\mathrm{P}<0.001)$ with ongoing lactation, stearic acid decreased $(\mathrm{P}<0.001)$ by LLS. But decreasing of oleic acid with lactation wasn't consistent with the reports of Pakistan and Norwegian goats $(9,41)$. Short chain fatty acids (from C4:0 to C10:0) were responsible for goat odours and were defined as rancid and tart $(9,39)$ and it was postulated that short chain fatty acids occurred from depletion of body fats (14). Our findings showed that short chain fatty acids increased with lactation duration because depletion of body fats probably increased and short chain fatty acids broke free. On the other hand, some breeders state that source of goat odour is oestrus. This opinion can explain why oestrus generally comes up at the last trimester of lactation and increases the depletion of body fats. Several previous studies on different goat breeds $(7,41,42)$ show that milk fat contains twenty-two carbon fatty acids as the longest chain (C22:0 and C22:6). Also, fatty acids with about twenty-four carbons (C24:0) were detected in Damascus goat milk in this study. The reason for this may be related to gas chromatography conditions and breed effect.

Sums and ratios based on fatty acids were generally shaken via feeding systems and it were not altered by age and birth types. Varied feed resources, especially browses and meadow plants, generate unequable ratios of unsaturated fatty acids (UFA) in milk in so far as increased UFA ratios or due to dissimilarity way of feed is finished in the rumen (35). The shape of rumen process for varied feed resources are different because of the different lipolysis procedures put accounted by rumen enzymes (21, 29). The detected SFA and UFA values were similar with numerous reports for different breeds $(3,7,35,41)$. SFA ratio in PBFG was lower than CBFG $(\mathrm{P}<0.001)$ and UFA ratio was higher in MLS $(\mathrm{P}<0.01)$. These results that depended on feeding systems in this study showed consistence with some earlier reports $(14,35)$. But the transition from SFA to UFA didn't occur in ELS and LLS. Results show that the effect of feeding systems on fatty acid ratios may be different among stages of lactation. Another index used to access the important value of fat is PUFA/SFA and the recommended value for the diet is 0.45 . High levels of PUFA/SFA are desired because this may induce an increase in low blood cholesterol levels $(8$, 36). In the present research, this percentage differs importantly among lactation stages $(\mathrm{P}<0.001)$. The values were found to be lower than recommended levels. The effect of fatty acids on sanitation is not exactly stated by the rates a forenamed. Thus, it is suggested that NV, AI and TI should be ciphered. NV refers the wellness of the 
feed in virtue of its lipid composition. AI values higher than 1 point to atherosclerosis risk and TI indicates potential aggregation of blood platelets; both are recommended for a healthy diet (44). The NV, AI and TI of PBFG yielded better values than CBFG in MLS $(\mathrm{P}<0.01 ; \mathrm{P}<0.001)$ because NV increased while AI and TI decreased. Although values of AI and TI in ELS and MLS were lower than the values found by Chiofalo et al. (5) for ewes, they were compatible with their indications in LLS. Goat odour in milk is a very important parameter for consumption of milk. If milk has goat odour, it may not be preferred by consumers. In the present study, OI was calculated first time for goat milk and results indicated that PBFG had significantly lower values than CBFG in MLS (5.891 means difference) $(\mathrm{P}<0.001)$ and LLS $(2.566$ means difference) $(\mathrm{P}<0.05)$. When the sums and ratios based on fatty acids were evaluated in terms of lactation stages, it was shown that all values significantly differed among stages of lactation. It was noted that all values deteriorated as lactation progressed.

In conclusion, pasture-based feeding system may be favored over concentrate based feeding systems for more milk production and better milk quality traits, but it should be noted that starting of pasture period after parturition should be acclimatized to exercise. On the other hand, it was shown that lactation duration was not affected by feeding systems. It was detected that milk SCC was not related to mastitis although it could tend to increase during lactation stages. As milk mineral matters decreased with ongoing lactation, it is advised that more mineral should be supplemented according to lactation periods for goat health and healthy milk production. The increase of short chain fatty acids towards the end of lactation showed the destruction of depot fats and hence goats should be fed a more balanced diet in this period. Also, it says that age and birth types generally were not important on milk quality parameters. According to these results, it can be argued that milk quality is generally better in ELS than LLS. Finally, feeding system based on pasture and the obtained milk in ELS may be more preferable compared to concentrate based feeding systems and LLS. Feeding programs should be organized according to lactation stages to ensure good milk quality parameters.

\section{Acknowledgement}

This study was financially supported by Mustafa Kemal University (Project Number: 10560).

\section{References}

1. Aganga AA, Amarteifio JO, Nkile N (2002): Effect of stage of lactation on nutrient composition of Tswana sheep and goat's milk. J Food Comp Anal, 15, 533-543.

2. Akcapinar H, Ozbeyaz C (1999): Hayvan Yetişstiriciliği Temel Bilgileri. Kariyer Matbaacılık, Ankara.
3. Ceballos LS, Morales ER, de la Torre Adarve G et al. (2009): Composition of goat and cow milk produced under similar conditions and analyzed by identical methodology. $\mathrm{J}$ Food Compos Anal, 22, 322-329.

4. Chemineau P, Gauthier D, Poirier JC, et al. (1982): Plasma levels of $\mathrm{LH}, \mathrm{FSH}$, prolactin, oestradiol-17 $\beta$ and progesterone during natural and induced oestrus in the dairy goat. Theriogenology, 17, 313-323.

5. Chiofalo B, Liotta L, Zumbo A, et al. (2004): Administration of olive cake for ewe feeding: effect on milk yield and composition. Small Rumin Res, 55, 169-176.

6. Das M, Singh M (2000): Variation in blood leucocytes, somatic cell count, yield and composition of milk of crossbred goats. Small Rumin Res, 35, 169-174.

7. Decandia M, Cabiddu A, Molle G, et al. (2007): Effect of different feeding systems on fatty acid composition and volatile compound content in goat milk. Options Mediterraneennes, Series A: Advanced Nutrition and Feeding Strategies to Improve Sheep and Goat Production. Centre International de Hautes Etudes Agronomique Méditerranéennes, Zaragoza, 129-134.

8. Department of Health (1994): Nutritional aspects of cardiovascular disease. Report on health and social subjects, No.46, HMSO, London.

9. Eknæs M, Havrevoll Ø, Volden H, et al. (2009): Fat content, fatty acid profile and off-flavours in goats milkeffects of feed concentrates with different fat sources during the grazing season. Anim Feed Sci Technol, 152, 112-122.

10. Ergun A, Tuncer SD, Colpan I, et al. (2006): Hayvan besleme ve beslenme hastalıkları. Pozitif matbaacilık, Ankara.

11. Erol H, Akcadag HI, Unal N, et al. (2012): Ankara keçilerinde süt verimi ve oğlaklarda büyümeye etkisi. Ankara Üniv Vet Fak Derg 59, 129-134.

12. Esterbauer H, Cheeseman CH (1990): Determination of aldehydic lipid peroksidation products: Malonaldehyde and 4-hydroxynonenal. Methods in Enzymology, 186, 407- 421.

13. Galina MA, Osnaya F, Cuchillo HM, et al. (2007): Cheese quality from milk of grazing or indoor fed Zebu cows and Alpine crossbred goats. Small Rumin Res, 71, 264-272.

14. Goetsch AL, Zeng SS, Gipson TA (2011): Factors affecting goat milk production and quality. Small Rumin Res, 101, 55-63.

15. Gomes V, Della Libera AMMP, Paiva M, et al. (2006): Effect of the stage of lactation on somatic cell counts in healthy goats (Caprae hircus) breed in Brazil. Small Rumin Res, 64, 30-34.

16. Gül S, Keskin M, Kaya Ş (2010): Olive cake usage as an alternative to cotton seed meal in dairy goat feeding. Afr $\mathrm{J}$ Agric Res, 5, 1643-1646.

17. Guler Z, Keskin M, Masatcioglu T, et al. (2007): Effects of Breed and Lactation Period on Some Characteristics and Free Fatty Acid Composition of Raw Milk from Damascus Goats and German Fawn x Hair Goat B1 Crossbreds. Turk J Vet Anim Sci, 31, 347-354.

18. Hinckley LS, Williams LF (1981): Diagnosis of mastitis in goats. Veterinary medicine, small animal clinician: VM, SAC, 76, 711.

19. Iriadam M (2004): Kilis keçilerine ait bazı hematolojik ve biyokimyasal parametreler. Ankara Üniv Vet Fak Derg, 51, 83-85. 
20. Kondyli E, Katsiari MC (2002): Fatty acid composition of raw caprine milk of a native Greek breed during lactation. Int J Dairy Technol, 55, 57-60.

21. Lee MRF, Winters AL, Scollan ND, et al. (2004): Plantmediated lipolysis and proteolysis in red clover with different polyphenol oxidase activities. Journal Sci Food Agric, 84, 1639-1645.

22. Min BR, Hart SP, Sahlu T, et al. (2005): The effect of diets on milk production and composition, and on lactation curves in pastured dairy goats. J Dairy Sci, 88, 2604-2615.

23. Morand-Fehr P, Fedele V, Decandia M, et al. (2007): Influence of farming and feding systems on composition and quality of goat and sheep milk. Small Rumin Res, 68, 2034.

24. Murray RK, Granner KD, Mayes AP, et al. (2004): Harper Biyokimya. Çeviri Edi. Nurten Dikmen, Tuncay Özgünen, Nobel Tip Kitabevleri, ISBN: 975- 420- 389- X, İstanbul.

25. Orman A, Gunay A, Balci F, et al. (2011): Monitoring of somatic cell count variations during lactation in primiparous and multiparous Turkish Saanen goats (Capra hircus). Turk J Vet Anim Sci, 35, 167-175.

26. Paape MJ, Wiggans GR, Bannerman DD, et al. (2007): Monitoring goat and sheep milk somatic cell counts. Small Rumin Res, 68, 114-125.

27. Park YW, Chukwu HI (1998): Macro-mineral concentrations in milk of two goat breeds at different stages of lactation. Small Rumin Res, 1, 157-166.

28. Park YW, Chukwu HI (1989): Trace mineral concentrations in goat milk from French-Alpine and AngloNubian breeds during the first 5 months of lactation. J Food Comp Anal, 2, 161-169.

29. Park YW, Juárez M, Ramos M, et al. (2007): Physicochemical characteristics of goat and sheep milk. Small Rumin Res, 68, 88-113.

30. Prasad H, Tewari HA, Sengar OPS (2005): Milk yield and composition of the beetal breed and their crosses with Jamunapari, Barbari and Black Bengal breeds of goat. Small Rumin Res, 58, 195-199.

31. Raynal-Ljutovac K, Lagriffoul G, Paccard P, et al. (2008): Composition of goat and sheep milk products: An update. Small Rumin Res, 79, 57-72.

32. Raynal-Ljutovac K, Pirisi A, De Cremoux R, et al. (2007): Somatic cells of goat and sheep milk: analytical, sanitary, productive and technological aspects. Small Rumin Res, 68, 126-144.

33. Rufián-Henares JÁ, Guerra-Hernandez E, GarcíaVillanova B (2006): Colour measurement as indicator for controlling the manufacture and storage of enteral formulas. Food Control, 17, 489-493.

34. Sampelayo MRS, Amigo L, Ares JL, et al. (1998): The use of diets with different protein sources in lactating goats: composition of milk and its suitability for cheese production. Small Rumin Res, 31, 37-43.
35. Sampelayo MRS, Pérez L, Martn Alonso JJ, et al. (2002): Effects of concentrates with different contents of protected fat rich in PUFAs on the performance lactating Granadina goats-Part II. Milk production and composition. Small Rumin Res, 43, 141-148.

36. Santos-Silva J, Bessa RJB, Santos-Silva F (2002): Effect of genotype, feeding system and slaughter weight on the quality of light lambs II. Fatty acid composition of meat. Livest Prod Sci, 77, 187-194.

37. Silanikove N, Leitner G, Merin U, et al. (2010): Recent advances in exploiting goat's milk: quality, safety and production aspects. Small Rumin Res, 89, 110-124.

38. Solah VA, Staines V, Honda S, et al. (2007): Measurement of milk color and composition: effect of dietary intervention on western Australian holstein-friesian cow's milk quality. J Food Sci, 72, 560-566.

39. Soryal K, Beyene FA, Zeng S, et al. (2005): Effect of goat breed and milk composition on yield, sensory quality, fatty acid concentration of soft cheese during lactation. Small Rumin Res, 58, 275-281.

40. Suriyasathaporn W, Vinitketkumnuen U, Chewonarin T, et al.(2006): Higher somatic cell counts resulted in higher malondialdehyde concentrations in raw cows' milk. Int Dairy J, 16, 1088-1091.

41. Talpur FN, Bhanger MI, Khooharo AA, et al. (2008): Seasonal variation in fatty acid composition of milk from ruminants reared under the traditional feeding system of Sindh. Pakistan Livest Sci,118, 166-172.

42. Talpur FN, Bhanger MI, Memon NN (2009): Milk fatty acid composition of indigenous goat and ewe breeds from Sindh, Pakistan. J Food Compos Anal, 22, 59-64.

43. TUIK (2015): Animal production statistics. Available at www. tuik.gov.tr. (Accessed January 15, 2018)

44. Ulbricht TL, Southgate DA (1991): Coronary heart disease: seven dietary factors. Lancet, 338, 985-992.

45. Yakan A, Ates CT, Alasahan S, et al. (2016): Damascus kids' slaughter, carcass and meat quality traits in different production systems using antioxidant supplementation. Small Rumin Res 2016; 136, 43-53.

46. Yakan A (2012): Record methods and calculation of lactation milk production in sheep and goat. AVKAE Derg, 2, 18-23.

47. Yang FL, Li XS, He BX, et al. (2011): Malondialdehyde level and some enzymatic activities in subclinical mastitis milk. Afr J Med Med Sci, 10, 5534-5538.

Geliş tarihi: 18.03.2018 / Kabul tarihi: 15.07.2018
Address for correspondence:
Prof. Dr. Necmettin ÜNAL
Ankara University, Veterinary Faculty
Department of Animal Breeding
06110, Ankara, Turkey
e-mail:unaln@ankara.edu.tr 